\title{
A NEW REALIZATION OF THE GLOBAL LUNAR REFERENCE FRAME BASED ON CO-REGISTERED LOLA TRACKS
}

\author{
P. Gläser ${ }^{1,2, *}$ I. Haase ${ }^{1}$, J. Oberst ${ }^{1,3}$ \\ ${ }^{1}$ Technical University Berlin, Dept. of Planetary Geodesy, 10623 Berlin, Germany - philipp.glaeser@tu-berlin.de \\ ${ }^{2}$ Ronin Institute for Independent Scholarship, Montclair, NJ 07043, USA \\ ${ }^{3}$ German Aerospace Center, Dept. of Planetary Geodesy, 12489 Berlin, Germany
}

Commission III, WG III/II

KEY WORDS: Moon, LOLA, Reference Frame, Co-Registration, Polar Radius

\begin{abstract}
:
We present a method that aligns lunar south and north pole LOLA DTMs using selected LOLA tracks and co-registration techniques. The selected LOLA tracks were then co-registered to the aligned polar DTMs with the aim to create a new LOLA frame of high relative accuracy. At the poles the relative accuracy of the resulting LOLA frame improved in comparison with the original LOLA frame, especially at the north pole. At lower latitudes on the lunar near side we could show that we achieve smaller residuals between our LOLA frame and a photogrammetrically derived reference DTM than with the original LOLA frame. On the far side we could not achieve better results which we believe is stemming from the generally less accurate orbit knowledge there. From the aligned polar DTMs we were able to derive a polar radius of $1738,049 \mathrm{~km}$.
\end{abstract}

\section{INTRODUCTION}

Physical measurements can only be compared or interpreted when the reference system in which the measurement was taken is known. There are well-defined and meaningful criteria describing how to create a reference system, e.g. the International Celestial Reference System (ICRS) (Souchay and Feissel-Vernier, 2006). A reference system refers to the description of how the system is set up, like origin, direction of axes etc., whereas a reference frame refers to the materialization of such system, e.g. a catalog of precise measurements substantiating the reference system.

For the coordinates on the lunar surface two distinct body-fixed reference systems are commonly used in the scientific community (Williams, 2008). The Mean Earth/Polar Axis (ME) reference system is based on the mean geometric orientation of the Moon with respect to Earth. Here, the z-axis is defined as the mean lunar rotational pole and the prime meridian $\left(0^{\circ}\right.$ longitude $)$ is defined by the mean Earth direction. In contrast, the Principal Axes (PA) reference system is based on the orientation of the moment-of-inertia (principal) axes. The PA and ME rotation axes do not coincide since the Moon is not truly a synchronously rotating triaxial ellipsoid. At the lunar surface coordinates given in the two systems can differ by about one kilometer, however, a constant, time-independent rotation can be applied to either of the systems to get to the other.

The ME system is the recommended system to be used for lunar mapping and is generally referred to when coordinates are exchanged (Archinal, 2006). For dynamical studies such as gravity field determination or Lunar Laser Ranging (LLR) the PA system is preferably used (Chapront and Francou, 2006). Both systems are basically defined by measurements to the Laser Ranging Retro Reflectors (LRRRs) located at three Apollo and two Lunokhod sites, which mark the only anchor points on the lunar surface.

\footnotetext{
${ }^{*}$ Corresponding author
}

Since the Apollo era several spacecraft orbited the Moon and carried out precise mapping of its surface morphology with laser altimeters, e.g. Clementine, Kaguya, Chang'E 1, Chandrayaan-1 and the Lunar Reconnaissance Orbiter (LRO). LRO, in particular, is equipped with the powerful Lunar Orbiting Laser Altimeter (LOLA) (Smith et al., 2017), which is the data set of choice for this study and currently provides the most accurate global geodetic grid. However, these observations are made from a fast moving spacecraft whose position and attitude comes with uncertainties (ten meter level) which propagate to the derived surface coordinate (Mazarico et al., 2013). In this work we show a method to relatively co-register LOLA tracks to derive an improved version of the global LOLA reference frame and compare it to the absolutely known LRRR coordinates.

\section{METHODOLOGY}

The polar orbit of LRO presents a unique opportunity for precision mapping of the lunar polar areas. Here, all orbits and hence LOLA ground tracks converge and form a dense point cloud, which enables the production of high-resolution DTMs of down to the five to ten meter level, as previously shown in (Gläser et al., 2017). However, at this high resolution, the remaining orbit errors and consequently errors in the relative positional accuracy of ground points become relevant. To overcome these errors, we previously presented a co-registration technique (Gläser et al., 2013) which greatly improved the relative accuracy. The purpose of co-registering LOLA tracks in our previous work was to derive polar DTMs of high relative consistency for illumination studies (Gläser et al., 2014, Gläser et al., 2017).

In this work, however, we aim at developing a technique to be used globally to derive a set of global LOLA tracks that are correctly positioned with respect to each other and best represent the lunar coordinate frame. The idea is to tie pole-to-pole spanning LOLA tracks to the previously created polar DTMs as depicted in Fig. 1. Note that LOLA ground tracks suffer from disruptions when crossing the terminator, the so-called 'LOLA 
anomaly' (Smith et al., 2017). This implies that the continuous part of most LOLA tracks intersects the north- or the south pole only but not both. Also, with LRO in a slightly eccentric frozen orbit (pericenter near the south pole), LOLA currently cannot range to northern latitudes. Before we can adjust the tracks
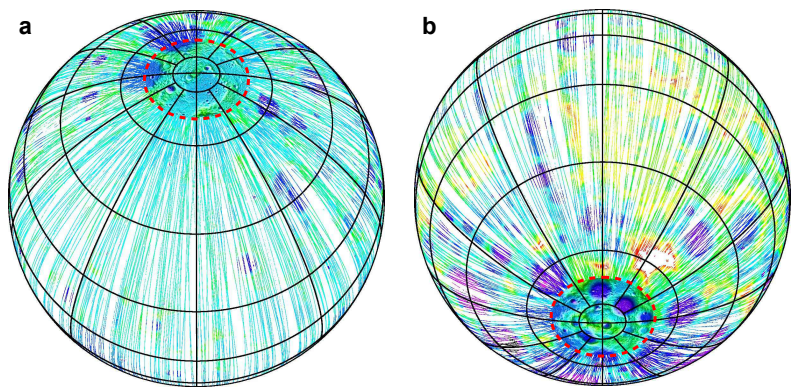

Figure 1. The pole-to-pole spanning LOLA tracks intersecting both DTMs (red circles) are shown in a general perspective view with grid lines drawn in $30^{\circ}$ longitudinal and $5^{\circ}$ latitudinal spacing and arctic circle at $88.5^{\circ} \mathrm{N} / \mathrm{S}$. Note that only $10 \%$ of the pole-to-pole spanning LOLA tracks are shown for reasons of visualization. (a) Northern hemisphere with north pole DTM. (b) Southern hemisphere with south pole DTM.

globally, we first needed to align the two independently created lunar polar DTMs to each other which then served as anchor points (control areas) for the coordinate frame. Therefore we selected that subset of the available $\sim 30,000$ LOLA tracks, that intersects both DTMs with at least 300 points each and with a standard deviation of height residuals of less than three meters. Then the DTMs were co-registered to this subset of ultimately 4,116 tracks and the common lateral and vertical offset for each DTM was determined. The offset in local stereographic map coordinates $x, y$ and height $h$ was found to be $1.45 \mathrm{~m}, 0.97 \mathrm{~m}$, $-0.01 \mathrm{~m}$ for the north pole and $2.48 \mathrm{~m},-1.63 \mathrm{~m},-0.06 \mathrm{~m}$ for the south pole. By applying the common offsets to the respective DTMs we have created polar DTMs that are registered to each other. However, the DTMs are only bedded in the bulk of LOLA tracks and each track itself can still have a remaining individual offset for the northern and the southern polar DTM. Now that the common offset between the subset of LOLA tracks and the two DTMs has been eliminated, the subset itself can be aligned to the DTMs. Hence, the 4,116 tracks were simultaneously co-registered to the two, now aligned, polar DTMs by adjusting the track location such that it best fits to both DTMs. Note, that LOLA tracks, which go once around the Moon passing over both poles, are about $10,916 \mathrm{~km}$ long (circumference of a sphere with a radius of $1737.4 \mathrm{~km}$ ) and only a few percent of the track actually intersect with the polar DTMs, typically $\sim 1-3 \%$. Consequently, only a small fraction of the track can be co-registered with the DTM where it fits well but has leeway otherwise. Although the total maximum intersection ratio of a track regarding our two DTM approach is only $\sim 6 \%$, the DTMs are located antipodal and hence greatly contribute to the rigidity of the global registration.

Contrary to our previous work where we adjusted LOLA tracks only locally at the poles using three parameters we now find the optimal seven parameter Helmert transformation for each LOLA track when co-registering to both polar DTMs allowing for three additional degrees of freedom:

$$
\mathbf{X}^{\text {new }}=\mathbf{X}^{0}+\mu \cdot \mathbf{R}(\omega, \phi, \kappa) \cdot \mathbf{X}^{\text {org }}
$$

where $\mathbf{X}^{\text {org }}$ is the original LOLA track, $\mathbf{X}^{0}$ is the translation of the origin, $\mu$ is the scaling factor and $\mathbf{X}^{\text {new }}$ is the resulting new LOLA track (vectors and matrix are shown in bold). $\mathbf{R}(\omega, \phi, \kappa)$ is the rotation matrix which using the small angle approximation simplifies to

$$
\left[\begin{array}{ccc}
1 & \kappa & -\phi \\
-\kappa & 1 & \omega \\
\phi & -\omega & 1
\end{array}\right]
$$

Here, the LOLA tracks are incrementally rotated by small angles $(\omega, \phi, \kappa)$ about the lunar X-, Y-, and Z-axis of the ME reference system and translations of the origin $\left(\mathbf{X}^{0}=\left[\begin{array}{lll}d x d y & d z\end{array}\right]^{T}\right)$ and different scales $(\mu)$ are applied leading to a new set of coordinates for each track. Each of the rotation angles $\omega, \phi, \kappa$ were chosen to only take three values, e.g. $-\omega, 0,+\omega$, and are scaled in such a way that the applied rotation corresponds to a displacement of the track of five meter in the DTM. The translation of the origin can vary over \pm 40 pixels in all three dimensions and scale can take the three values $0.999998,1.0$ and 1.000002 . We chose the variation of the parameters in such a way since we expect the scale and rotation influence to be small compared to the shift of the origin. Finally, a grid search of the parameter space is performed which evaluates 81 translations and three rotation in each dimension and three scales leading to 43,046,721 different combinations. At each combination of parameters we calculate the standard deviation of the residuals of the LOLA profile with the DTMs which at the end of the process reveals which set of scale, translation and angles leads to the best fit between the track and the DTMs. Centered at the previously determined set of parameters a subsequent search with smaller (half) step-sizes follows to refine the result for Helmert transformation parameters. The algorithm is implemented for General Purpose Computation on Graphics Processing Unit (GPGPU) using OpenCL (Gaster et al., 2011) which runs the grid search in parallel on the GPU. The benefit of this approach in comparison to the one shown in (Gläser et al., 2013) is that we now determined a set of seven parameters, $d x, d y, d z, \omega, \phi, \kappa$ and $\mu$ which describes the best (global) fit of a track to both polar DTMs at the same time.

\section{RESULTS}

For our study we created two polar DTMs of ten meter per pixel resolution and with an extent of 200 by $200 \mathrm{~km}$ centered on the poles using co-registration techniques (Gläser et al., 2013). Each DTM was then co-registered with the common 4,116 LOLA tracks and the resulting offset was applied to ensure global alignment between them (Figures 1, 2).

\subsection{Polar radius}

Now that the DTMs are relatively aligned to each other we were able to derive a polar radius. The height values (relative to the mean lunar sphere of $1737.4 \mathrm{~km}$ ) of the DTM pixels containing the poles are $7.42 \mathrm{~m}$ for the north pole and $1291.06 \mathrm{~m}$ for the south pole. We added the average of both heights, $0.649 \mathrm{~km}$, to the radius of the lunar sphere leading to a polar radius of 1738.049 

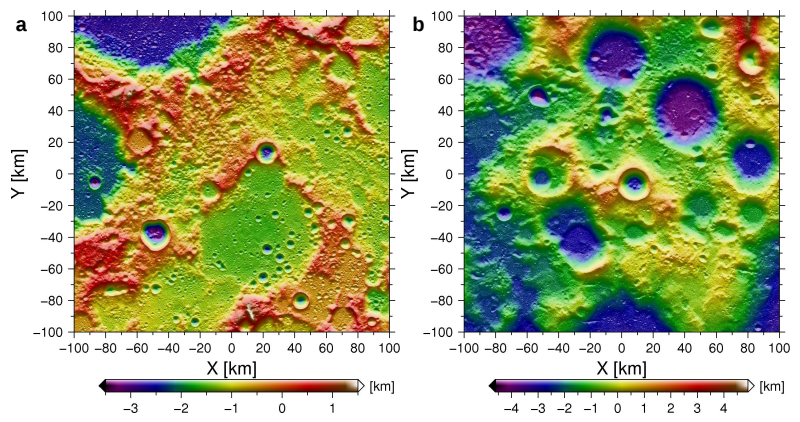

Figure 2. The LOLA DTMs have a resolution of ten meter per pixel, span 200 by $200 \mathrm{~km}$ and are displayed in polar stereographic projection. (a) North pole. (b) South pole.

$\mathrm{km}$. Our derived polar radius is larger than the average lunar radius of $1737.4 \mathrm{~km}$ which is due to the significant $c_{4,0}$-term (Smith et al., 2010).

\subsection{Frame characteristics}

The 4,116 common LOLA tracks were then simultaneously coregistered to the aligned polar DTMs. Only tracks that have a standard deviation of height residuals of less than three meters were considered. As a consequence, a total of 79 tracks were filtered out leading to 4,024 LOLA tracks, referred to as new LOLA frame, which consist of a total of 2,086,746,483 individual ground shots. At the equator the average spacing of the new LOLA frame is $2.7 \mathrm{~km}$ with a maximum gap of $31.47 \mathrm{~km}$ at $92.718^{\circ}$ longitude. At a latitude of $\pm 45^{\circ}$ the average spacing is $1.93 \mathrm{~km}$ and $1.92 \mathrm{~km}$ for the northern and southern hemisphere, respectively. To get an estimate on the expected global relative accuracy of our new LOLA frame we determined the residuals to the polar DTMs. Further we also determined the residuals of the corresponding original LOLA tracks, referred to as original LOLA frame, to the polar DTMs. By using the same orbits in the original and the new LOLA frame we make our results comparable. In Table 1 we show the standard deviations of the residuals achieved with the two LOLA frames when co-registered to the polar DTMs, indicating that we could homogenize and improve the residuals with our new LOLA frame. Note, that only tracks with residuals within two sigma of the data with more than 300 co-registered points and a standard deviation of height residuals of less than three meters were considered in the analysis. It is noticeable that the accuracy of the original LOLA frame at the south pole is comparable to our new LOLA frame, whereas great improvements (factor 2) could be achieved laterally for the north polar area. We suspect this is due to a generally better a-priori orbit knowledge for the south polar than the north polar regions due to a much larger data set and hence better orbit constraints using altimetric crossovers (Mazarico et al., 2012). With our new LOLA frame we achieve uniform standard deviations in $x, y$ and at both poles which are smaller than using the original LOLA frame. We expect these accuracies to be propagated along the ground tracks to equatorial latitudes as well.

\subsection{Global relative accuracy}

To test our assumption that the relative accuracy that we achieved at the poles is propagated along the ground tracks we coregistered the new LOLA frame to two lower latitudes DTMs. We determined the residual displacements of the tracks from our new LOLA frame to two photogrammetrically derived reference DTMs located at the near and far side, respectively. Similarly,

\begin{tabular}{|r|c|c|}
\hline & $\begin{array}{c}\text { Original } \\
\text { LOLA frame }\end{array}$ & $\begin{array}{c}\text { New } \\
\text { LOLA frame }\end{array}$ \\
\hline North Pole & {$[\mathrm{m}]$} & {$[\mathrm{m}]$} \\
$\sigma_{x}$ & 10.39 & 5.16 \\
$\sigma_{y}$ & 12.15 & 5.48 \\
$\sigma_{h}$ & 0.41 & 0.34 \\
\hline \hline South Pole & {$[\mathrm{m}]$} & {$[\mathrm{m}]$} \\
$\sigma_{x}$ & 6.60 & 5.38 \\
$\sigma_{y}$ & 5.55 & 5.25 \\
$\sigma_{h}$ & 0.31 & 0.34 \\
\hline
\end{tabular}

Table 1. Standard deviations of residual displacements of the original LOLA frame (left) and the new LOLA frame (right) with the north pole and south pole LOLA DTM. Values are given in meters in polar stereographic map coordinates $x, y$ and height $h$.

we also co-registered the original LOLA frame to these reference DTMs. The comparison of the residual displacements of tracks from the new with the original LOLA frame tracks reveals whether and to what extent the relative alignment improved. As reference we chose Kaguya Terrain Camera (TC) DTMs (Fig. 3) since DTMs derived from stereo imagery generally have a high relative accuracy and are ideal to check the track-to-track alignment.
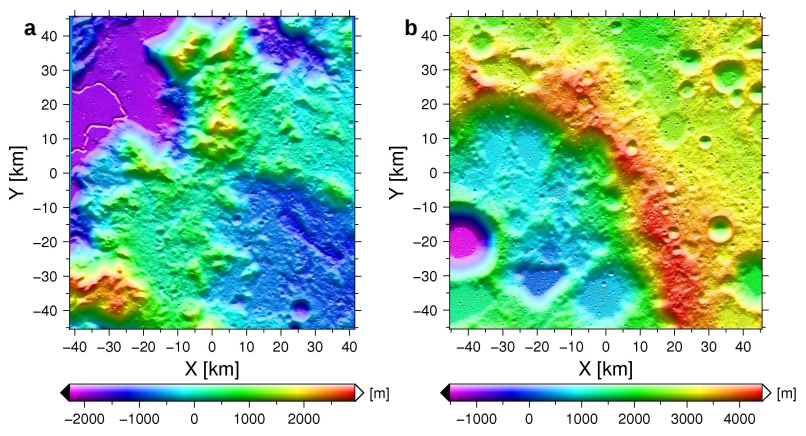

Figure 3. The TC DTMs have a resolution of $10 \mathrm{~m} / \mathrm{pixel}$ and are shown in local stereographic projection. (a) Near side: DTM is centered at $4.5^{\circ}$ longitude and $25.5^{\circ}$ latitude with the Apollo 15

landing site in the upper left corner. (b) Far side: DTM is centered at $181.5^{\circ}$ longitude and $-1.5^{\circ}$ latitude.

The first DTM (Fig. 3a), referred to as TC1 DTM, is centered at $4.5^{\circ}$ longitude and $25.5^{\circ}$ latitude showing the terrain of the Apollo 15 landing site in the upper left quadrant and spans 80 by $90 \mathrm{~km}$. The Apollo 15 landing site is located on the near side of the Moon where LRO's orbit can be observed directly from Earth and precisely reconstructed LOLA ground tracks are expected. In total 191 LOLA tracks intersect the TC1 DTM of which 74 orbits are also part of the new LOLA frame. We co-registered those 74 tracks, once taken from the new and once taken from the original LOLA frame, to the TC1 DTM and determined the residuals for each frame (Fig. 4). Tracks were considered outliers if they had less than 50 registered points, had a standard deviation of height residuals greater than seven meters or their residual shifts were not within two sigma of the data. In total 68 tracks were considered from the original LOLA frame and 67 from the new LOLA frame.

The second DTM (Fig. 3b), referred to as TC2 DTM, is centered at $181.5^{\circ}$ longitude and $-1.5^{\circ}$ latitude on the lunar far side and covers 90 by $90 \mathrm{~km}$. Here LRO's orbit is not backed by actual observations of the spacecraft and reconstructed LOLA ground 

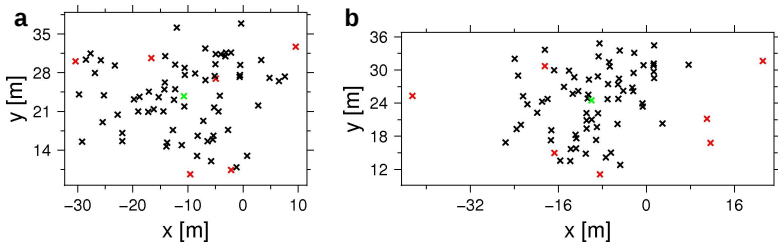

Figure 4. The residual shifts in local stereographic map coordinates $x, y$ of the 74 LOLA tracks to the TC1 DTM from

Fig. 3a. Considered residuals are shown as black crosses, outliers are shown as red crosses and the common offset between the DTM and all tracks is shown as a green cross. (a) Original LOLA frame. (b) New LOLA frame.

tracks are expected to be less precise. From the available 278 tracks 79 orbits are also part of the new LOLA frame. We coregistered these orbits from each of the two LOLA frames to the TC2 DTM, filtered outliers and determined the residuals for each data set (Fig. 5). In total 75 tracks were considered regarding the original LOLA frame and 73 tracks regarding the new LOLA frame.
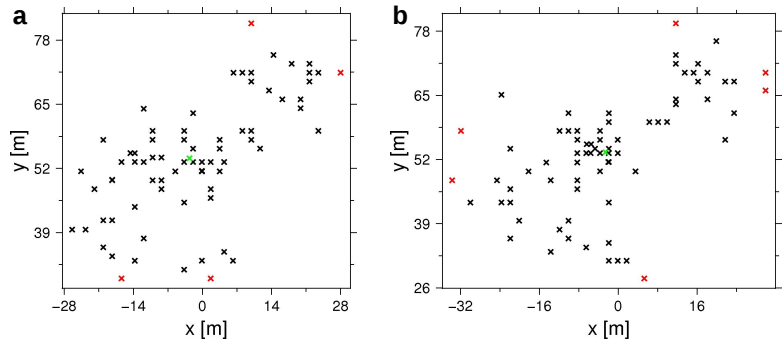

Figure 5. The residual shifts in local stereographic map coordinates $x, y$ of the 79 LOLA tracks to the TC2 DTM from

Fig. 3b. Considered residuals are shown as black crosses, outliers are shown as red crosses and the common offset between the DTM and all tracks is shown as a green cross. (a) Original LOLA frame. (b) New LOLA frame.

From the residuals (Figures 4 and 5) we determined the standard deviation for each LOLA frame. Additionally we also determined the standard deviation when using all available LOLA tracks in that area (see Table 2).

\begin{tabular}{|r|c|c||c|}
\hline & $\begin{array}{c}\text { Original } \\
\text { LOLA frame }\end{array}$ & $\begin{array}{c}\text { New } \\
\text { LOLA frame }\end{array}$ & $\begin{array}{c}\text { All } \\
\text { available }\end{array}$ \\
\hline $\mathrm{TC} 1$ & {$[\mathrm{~m}]$} & {$[\mathrm{m}]$} & {$[\mathrm{m}]$} \\
$\sigma_{x}$ & 9.30 & 7.67 & 7.83 \\
$\sigma_{y}$ & 6.26 & 5.96 & 6.67 \\
$\sigma_{h}$ & 1.08 & 1.14 & 1.09 \\
\hline \hline $\mathrm{TC} 2$ & {$[\mathrm{~m}]$} & {$[\mathrm{m}]$} & {$[\mathrm{m}]$} \\
$\sigma_{x}$ & 13.19 & 12.89 & 11.98 \\
$\sigma_{y}$ & 10.88 & 11.09 & 10.77 \\
$\sigma_{h}$ & 0.44 & 0.45 & 0.50 \\
\hline
\end{tabular}

Table 2. Standard deviations of residual displacements of the original LOLA frame (left), the new LOLA frame (middle) and all available LOLA tracks (right) for the near and far side reference DTMs TC1 and TC2. Values are given in meters in local stereographic map coordinates $x, y$ and height $h$.

When comparing the residuals between the TC1 DTM to the new and to the original LOLA frame it can be seen that we were able to achieve smaller standard deviations in the local stereographic $x$ - (east-west) and $y$-coordinates (north-south) and a similar standard deviation in $h$ (height). The standard deviations in $x$ and $y$ are also smaller when comparing the new LOLA frame to the case when all available LOLA tracks were co-registered. In general, all values at TC1 are below ten meters and hence are in good agreement with the Root Mean Square (RMS) values given for LRO's orbit overlaps (Mazarico et al., 2013) which as a first approximation are an estimate for the relative accuracy of the LOLA ground tracks. The level of accuracy achieved at the polar DTMs as well as more uniform standard deviations in $x, y$ were reproduced at TC1.

At TC2 we achieved a smaller standard deviation in $x$, a greater standard deviation in $y$ and a similar standard deviation in $h$ when comparing the new to the original LOLA frame. Compared to all available LOLA tracks we could not improve the lateral residuals and achieved similar but yet larger values. Although standard deviations in $x, y$ are more uniform for the new LOLA frame the level of accuracy as achieved at the polar DTMs cannot be reproduced at TC2. Here, the standard deviations in $x$ and $y$ are above ten meters for all three cases. We believe this might be due to a generally less accurate orbit knowledge for far side locations which cannot be corrected for with our approach.

\subsection{Absolute accuracy}

To investigate the absolute accuracy of the new LOLA frame, we used ortho-rectified LROC NAC (Lunar Reconnaissance Orbiter Camera Narrow Angle Camera) images at the $0.5 \mathrm{~m}$ pixel scale of three of the five LRRR sites: Apollo 11, 14 and Lunokhod 2 landing sites. The Lunokhod rover carried a laser reflector and at the Apollo sites astronauts installed LRRRs which are captured in the high resolution NAC images. The three LRRRs have been routinely observed through laser ranging and have coordinates known at the cm-level (Chapront and Francou, 2006). We processed two meter scale LROC NAC DTMs and ortho-images at half meter scale and then co-registered them to the original and the new LOLA frame. For comparison we measured the MEcoordinates of the LRRRs in the respective ortho-images of the co-registered NAC DTMs and compared them to the coordinates given in the literature (Williams, 2008). The distances retrieved

\begin{tabular}{|r|c|c||c|}
\hline & $\begin{array}{c}\text { Original } \\
\text { LOLA frame }\end{array}$ & $\begin{array}{c}\text { New } \\
\text { LOLA frame }\end{array}$ & $\begin{array}{c}\text { All } \\
\text { available }\end{array}$ \\
\hline Distance & {$[\mathrm{m}]$} & {$[\mathrm{m}]$} & {$[\mathrm{m}]$} \\
Apollo 11 & 9.10 & 7.53 & 6.65 \\
Apollo 14 & 7.83 & 7.15 & 6.63 \\
Lunokhod 2 & 7.90 & 9.92 & 11.27 \\
\hline
\end{tabular}

Table 3. The horizontal distances in meters between the LRRR in the NAC DTM and the observed coordinates through lunar laser ranging.

using the original LOLA frame and the new LOLA frame differ by a maximum of one DTM pixel (two meter), which is larger than the precision $(50 \mathrm{~cm})$ with which we can measure the LRRR site in the ortho-images. Within our measurement uncertainties the distances improved for the Apollo sites but degraded at the Lunokhod site (see Table 3). Nevertheless, we achieved similar results and could not show that global accuracy improved. Similar results are also achieved when comparing our results to the distances of the LRRRs in the NAC DTMs co-registered using all available LOLA tracks, see the third column of Table 3. Generally all three LOLA sets fit well to the absolute coordinate frame 
given that the distances are comparable to the RMS of orbit overlaps (Mazarico et al., 2013).

\section{SUMMARY AND CONCLUSION}

We connected and aligned the locally adjusted lunar polar LOLA DTMs via the co-registration of 4,116 pole-to-pole spanning tracks. The aligned DTMs allowed us to derive a lunar polar radius. Subsequently, we successfully co-registered the 4,116 tracks simultaneously to both DTMs with our proposed method leading to 4,024 tracks for our new LOLA frame after filtering. We showed that the new LOLA frame has smaller residuals with the polar DTMs than the original LOLA frame and hence a higher relative accuracy. To verify that the tracks are properly located on a global scale we co-registered them to two Kaguya TC DTMs, one located on the near and one on the far side. We demonstrated that the standard deviations of the residual shifts of our LOLA frame are comparable to the original LOLA frame regarding the far side TC DTM and in the case of the near side TC DTM smaller than the original LOLA frame. We argue that the point-to-point accuracy of tracks on the near side is higher due to direct observations of the spacecraft, which once the track is anchored at the poles the track-to-track accuracy is delivered to lower latitudes as well. Nevertheless the original LOLA frame has such a high inner accuracy already that the improvements are small regarding a global scale. Through analysis of the position of three LRRRs (Apollo 11, Apollo 14, Lunokhod 2) we were able to show that our frame fits well to the only known absolute coordinates on the Moon. There is no evidence, however, that our new LOLA frame has a higher absolute accuracy than the original LOLA frame.

\section{OUTLOOK}

The preliminary results gained with the proposed method are promising but our findings need more examples to undermine the made assumptions and to get solid statistics on the residuals. To improve the absolute coordinate knowledge of the new LOLA frame a global rotation of the polar DTMs along with the new LOLA frame could be carried out in order to fit to the coordinates of the LRRR sites.

\section{ACKNOWLEDGEMENTS}

P. Gläser was funded by a grant of the German Research Foundation (GL 865/2-1) and I. Haase by the Federal Ministry for Economic Affairs and Energy (FKZ 50OW1703). We also gratefully acknowledge the support of NVIDIA Corporation with the donation of the Quadro M5000 GPU used for this research.

\section{REFERENCES}

Archinal, B. A., 2006. Lunar Coordinates and Cartography: Coordinate System Establishment, Improvement, and Control (Registration) of Lunar Datasets, from Past, Present and Future U. S. and Foreign Missions. USGS Astrogeology Science Center pp. 119.

Chapront, J. and Francou, G., 2006. Lunar Laser Ranging: measurements, analysis, and contribution to the reference systems. IERS Technical Note 34, pp. 97-116.

Gaster, B., Howes, L., Kaeli, D. R., Mistry, P. and Schaa, D., 2011. Heterogeneous Computing with OpenCL. 1st edn, Morgan Kaufmann Publishers Inc., San Francisco, CA, USA.
Gläser, P., Haase, I., Oberst, J. and Neumann, G. A., 2013. Coregistration of laser altimeter tracks with digital terrain models and applications in planetary science. Planetary and Space Science 89, pp. 111-117.

Gläser, P., Oberst, J., Neumann, G., Mazarico, E., Speyerer, E. and Robinson, M., 2017. Illumination conditions at the lunar poles: Implications for future exploration. Planetary and Space Science.

Gläser, P., Scholten, F., De Rosa, D., Marco Figuera, R., Oberst, J., Mazarico, E., Neumann, G. A. and Robinson, M. S., 2014 Illumination conditions at the lunar south pole using high resolution Digital Terrain Models from LOLA. Icarus 243, pp. 78-90.

Mazarico, E., Goossens, S. J., Lemoine, F. G., Neumann, G. A., Torrence, M. H., Rowlands, D. D., Smith, D. E. and Zuber, M. T., 2013. Improved Orbit Determination of Lunar Orbiters with Lunar Gravity Fields Obtained by the GRAIL Mission. In: Lunar and Planetary Science Conference, Lunar and Planetary Science Conference, Vol. 44, p. 2414.

Mazarico, E., Rowlands, D. D., Neumann, G. A., Smith, D. E., Torrence, M. H., Lemoine, F. G. and Zuber, M. T., 2012. Orbit determination of the Lunar Reconnaissance Orbiter. Journal of Geodesy 86, pp. 193-207.

Smith, D. E., Zuber, M. T., Neumann, G. A., Lemoine, F. G., Mazarico, E., Torrence, M. H., McGarry, J. F., Rowlands, D. D., Head, J. W., Duxbury, T. H., Aharonson, O., Lucey, P. G., Robinson, M. S., Barnouin, O. S., Cavanaugh, J. F., Sun, X., Liiva, P., Mao, D.-d., Smith, J. C. and Bartels, A. E., 2010. Initial observations from the Lunar Orbiter Laser Altimeter (LOLA). Geophysical Research Letters 37, pp. L18204.

Smith, D. E., Zuber, M. T., Neumann, G. A., Mazarico, E., Lemoine, F. G., Head, III, J. W., Lucey, P. G., Aharonson, O., Robinson, M. S., Sun, X., Torrence, M. H., Barker, M. K., Oberst, J., Duxbury, T. C., Mao, D., Barnouin, O. S., Jha, K., Rowlands, D. D., Goossens, S., Baker, D., Bauer, S., Gläser, P., Lemelin, M., Rosenburg, M., Sori, M. M., Whitten, J. and Mcclanahan, T., 2017. Summary of the results from the lunar orbiter laser altimeter after seven years in lunar orbit. Icarus 283, pp. 70-91.

Souchay, J. and Feissel-Vernier, M., 2006. The international celestial reference system and frame. ICRS Center Report for 20012004 (IERS Technical Note ; 34).

Williams, J. G., 2008. DE421 lunar orbit, physical librations, and surface coordinates. 